\title{
Retraction Note: Mathematical Model of Hybrid and Electric Cars Control System
}

\author{
Vladimir Kozlovsky
}

Published online: 09 November 2021

Retracted article: E3S Web of Conferences 320, 01014 (2021)

https://doi.org/10.1051/e3sconf/202132001014

This paper has been formally retracted from publication by the author on ethical grounds. Actually, a misunderstanding occurred between a few authors, including Vladimir Kozlovsky, with the result that they mistakenly sent the same article simultaneously to two different conferences. The author is responsible for this mistake and apologise for it. Request approved by the proceedings Editor and the Publisher on November 17, 2021. 